\title{
Article \\ An Active Set Limited Memory BFGS Algorithm for Machine Learning
}

\author{
Hanger Liu ${ }^{1}$, Yan $\mathrm{Li}^{2, *}$ and Maojun Zhang ${ }^{3, *(D)}$ \\ 1 Center for Applied Mathematics of Guangxi, College of Mathematics and Information Science, \\ Guangxi University, Nanning 530004, China; 2006301023@st.gxu.edu.cn \\ 2 School of Mathematics and Statistics, Baise University, Baise 533000, China \\ 3 School of Business, Suzhou University of Science and Technology, Suzhou 215011, China \\ * Correspondence: lybsxy@163.com (Y.L.); 2721@mail.usts.edu.cn (M.Z.)
}

check for

updates

Citation: Liu, H.; Li, Y.; Zhang, M. An Active Set Limited Memory BFGS Algorithm for Machine Learning. Symmetry 2022, 14, 378. https:// doi.org/10.3390/sym14020378

Academic Editor: Aviv Gibali

Received: 21 December 2021

Accepted: 17 January 2022

Published: 14 February 2022

Publisher's Note: MDPI stays neutral with regard to jurisdictional claims in published maps and institutional affiliations.

Copyright: () 2022 by the authors Licensee MDPI, Basel, Switzerland. This article is an open access article distributed under the terms and conditions of the Creative Commons Attribution (CC BY) license (https:// creativecommons.org/licenses/by/ $4.0 /)$.

\begin{abstract}
In this paper, a stochastic quasi-Newton algorithm for nonconvex stochastic optimization is presented. It is derived from a classical modified BFGS formula. The update formula can be extended to the framework of limited memory scheme. Numerical experiments on some problems in machine learning are given. The results show that the proposed algorithm has great prospects.
\end{abstract}

Keywords: nonconvex stochastic optimization; stochastic approximation; quasi-Newton method; damped limited-memory BFGS method; variance reduction

PACS: 62L20; 90C30; 90C15; 90C60

\section{Introduction}

Machine learning is an interdisciplinary subject involving probability theory, statistics, approximation theory, convex analysis, algorithm complexity theory, and so on. In machine learning, people usually construct an appropriate model from an extraordinary large amount of data. Therefore, the traditional algorithms for solving optimization problems are no longer suitable for machine learning problems. A stochastic algorithm must be used to solve the model optimization problem we encounter in machine learning.

This type of problem is considered in machine learning

$$
\min _{x \in \Re^{d}} f(x)=\mathbb{E}[T(x, \tau)]
$$

where $T: \Re^{D_{x}} \times \Re^{n} \rightarrow \Re$ is a continuously differentiable function, $\mathbb{E}[\cdot]$ denotes the expectation taken with respect $\tau$ and $\tau$ is the random variable of the distribution function $\mathbb{P}$. In most practical cases, the function $T(\cdot, \tau)$ is not given intuitively. In addition, even worse, the distribution function $\mathbb{P}$ may also be unknown. The objective function (1) is defined using the empirical expectation

$$
f(x)=\frac{1}{\mathbb{N}} \sum_{n=1}^{\mathbb{N}} f_{i}(x),
$$

where $f_{i}: \Re^{D_{x}} \rightarrow \Re$ is the loss function that corresponds to the ith data sample, and $\mathbb{N}$ denotes the number of data samples which is assumed to be extremely large.

The stochastic approximation $(S A)$ algorithm is usually used to solve the above problems by Robbins and Monro [1]. The original SA algorithm can also be called random gradient descent $(S G D)$. It is somewhat similar to the classical steepest descent method, which adopts the iterative process of $x_{k+1}=x_{k}-\alpha_{k} g_{k}$. In general, random gradient $g_{k}$ is used to represent the approximation of the full gradient $\nabla f$ of $f$ at $x_{k}$ and $\alpha_{k}$ is the step size (Learning rate). The SA algorithm has been deeply studied by many scholars [2-4]. 
In this thesis, we mainly study the stochastic second-order method, that is, stochastic quasi-Newton methods $(S Q N)$ to solve problem (2). Among the traditional optimization methods, the quasi-Newton methods have faster convergence speed and higher convergence accuracy than the first-order method because it uses the approximate second-order derivative information. The quasi Newton method is usually updated by the following iterative formula:

$$
x_{k+1}=x_{k}-\alpha_{k} B_{k}^{-1} \nabla f(x) \quad \text { or } \quad x_{k+1}=x_{k}-\alpha_{k} H_{k} \nabla f(x)
$$

where $B_{k}$ is the symmetric positive definite approximation of Hessian matrix $\nabla^{2} f(x)$ at $x_{k}$ or $H_{k}$ is the symmetric positive definite approximation of $\left[\nabla^{2} f(x)\right]^{-1}$. In the traditional BFGS algorithm, the iterative formula of $B_{k}$ is as follows:

$$
B_{k}=B_{k-1}+\frac{y_{k-1} y_{k-1}^{T}}{s_{k-1}^{T} s_{k-1}}-\frac{B_{k-1} s_{k-1} s_{k-1}^{T} B_{k-1}}{s_{k-1}^{T} B_{k-1} s_{k-1}},
$$

where $s_{k-1}=x_{k}-x_{k-1}=\alpha_{k} d_{k}$ and $y_{k-1}=\nabla f\left(x_{k}\right)-\nabla f\left(x_{k-1}\right)$. If formula ShermanMorrison-Woodbury formula is used, the iterative formula of $H_{k}$ can be easily obtained:

$$
H_{k}=\left(I-\frac{s_{k-1} y_{k-1}^{T}}{s_{k-1}^{T} y_{k-1}}\right) H_{k-1}\left(I-\frac{s_{k-1} y_{k-1}^{T}}{s_{k-1}^{T} y_{k-1}}\right)+\frac{s_{k-1} s_{k-1}^{T}}{s_{k-1}^{T} y_{k-1}} .
$$

It is very important to use a limited memory variant for large-scale problems. This so-called L-BFGS [5] algorithm has a linear convergence rate. It produces well scaled and productive search directions that yield an approximate solution in fewer iterations and function evaluations. In stochastic optimization, many stochastic quasi Newton formulas have been proposed.

The LBFGS method has the following iteration rule $x_{k+1}=x_{k}-\alpha_{k} H_{k} \nabla f(x)$. The LBFGS method updates $H_{k}$ by the following rule:

$$
\begin{aligned}
H_{k} & =Q_{k-1}^{T} H_{k-1} Q_{k-1}+\rho_{k-1} s_{k-1} s_{k-1}^{T} \\
& =Q_{k-1}^{T}\left[Q_{k-2}^{T} H_{k-2} Q_{k-2}+\rho_{k-2} s_{k-2} s_{k-2}^{T}\right] Q_{k-1}+\rho_{k-1} s_{k-1} s_{k-1}^{T} \\
& =\ldots \\
& =\left[Q_{k-1}^{T} \ldots Q_{k-r+1}^{T}\right] H_{k-r+1}\left[Q_{k-r+1}^{T} \ldots Q_{k-1}^{T}\right] \\
& +\rho_{k-r+1}\left[Q_{k-2}^{T} \ldots Q_{k-r+2}^{T}\right] s_{k-r+1} s_{k-r+1}^{T}\left[Q_{k-r+2}^{T} \ldots Q_{k-2}^{T}\right] \\
& +\cdots+\rho_{k-1} s_{k-1} s_{k-1}^{T},
\end{aligned}
$$

where $Q_{k-1}=I-\rho_{k-1} y_{k-1} s_{k-1}^{T}, \rho_{k-1}=\frac{1}{s_{k-1}^{T} y_{k-1}}$ and $r$ is the memory size. Bordes, Bottomu, and Gallinari studied the quasi Newton method of diagonal rescaling matrix based on secant in [6]. In [7], Byrd et al. proposed a stochastic LBFGS method based on SA and proved its convergence for strongly convex problems. In [8], Gower, Goldfarb, and Richtárik proposed a variance reduced block L-BFGS method that converges linearly for convex functions. It is worth noting that, in the above quasi-Newton methods, the convergence of the algorithm needs to be convex or strongly convex.

If the objective function itself does not have the property of convexity, there are several problems that the LBFGS method has difficulty overcoming:

- How can we guarantee the positive definiteness of iterative matrix $H_{k}$ without line search?

- How can we guarantee the convergence of the proposed L-BFGS method?

These problems seem particularly difficult. However, a modified stochastic limited BFGS (LMLBFGS) is proposed to solve the above problems. On this basis, a new improved 
algorithm (LMLBFGS-VR) is proposed. Note that our presented algorithm can be adapted to approximate the solution of a nonlinear system of equations in [9].

This paper is divided into five parts: in Section 2, the LMLBFGS and LMLBFGS-VR are presented and their convergence properties are discussed in Section 3. In Section 4, the numerical experiments of the proposed algorithm are given. A summary is given in the last part.

\section{Premise Setting and Algorithm}

In this part, a new LBFGS(LMLBFGS) algorithm is proposed, which can automatically generate a positive definite matrix $B_{k}$.

\subsection{LMLBFGS Algorithm}

In order to solve this kind of problem, suppose that $\mathscr{E} \subset \Re^{n}$ does not depend on $x$ and the random gradient $g(x, \tau)$ at $x$ is generated by a stochastic first-order oracle (SFO), for which the distribution of $\tau$ is supported on $\mathscr{E} \subset \Re^{n}$. It is common to use a mini-batch stochastic gradient of the $i$-th sampling during the $k$-th iteration, which is described as

$$
g_{k}=\frac{1}{z_{k}} \sum_{i \in Z_{k}} g\left(x_{k}, \tau_{k, i}\right)=\frac{1}{z_{k}} \sum_{i \in Z_{k}} \nabla f_{i}\left(x_{k}\right),
$$

and a sub-sampled Hessian defined as follows

$$
G_{k}=\frac{1}{z_{k}^{*}} \sum_{i \in Z_{k}^{*}} G\left(x_{k}, \tau_{k, i}\right)=\frac{1}{z_{k}^{*}} \sum_{i \in Z_{k}^{*}} \nabla^{2} f_{i}\left(x_{k}\right) .
$$

We have the subset $Z_{k}$ and $Z_{k}^{*}$ is the sample number where $z_{k}$ and $z_{k}^{*}$ are the cardinalities of $Z_{k}$ and $Z_{k}^{*}$. $\tau_{k, i}$ is a random variable. From the definition of random gradient, it is not difficult to find that the random gradient under this setting can be calculated faster than the full gradient. We assume here that the SFO generation method can separate $x_{k}$ and $\tau_{k}$ independently and generate the output $g\left(x_{k}, \tau_{k, i}\right)$. Therefore, the stochastic gradient difference and the iterative difference are defined as

$$
\begin{gathered}
y_{k}=g_{k}-g_{k-1}=\frac{1}{z_{k}} \sum_{i \in Z_{k}} g\left(x_{k}, \tau_{k, i}\right)-\frac{1}{z_{k-1}} \sum_{i \in Z_{k-1}} g\left(x_{k-1}, \tau_{k-1, i}\right), \\
s_{k}=x_{k}-x_{k-1} .
\end{gathered}
$$

In traditional methods, the authors in [10] proposed a new type of $\overline{y_{k}}$ by using

$$
\overline{y_{k}}=y_{k}+\lambda_{k} s_{k}
$$

where

$$
\lambda_{k}=\frac{2\left[f\left(x_{k-1}\right)-f\left(x_{k}\right)\right]+\left(g_{k}+g_{k-1}\right)^{T} s_{k}}{\left(s_{k}^{T} y_{k}\right)^{2}} \cdot\left(y_{k} y_{k}^{T}\right) .
$$

Inspired by their methods, we have the following new definitions:

$$
y_{k}^{*}=y_{k}+\lambda_{k} s_{k}
$$

where

$$
\lambda_{k}=\frac{2\left[f\left(x_{k-1}\right)-f\left(x_{k}\right)\right]+\left(g_{k}+g_{k-1}\right)^{T} s_{k}}{\max \left\{\left(s_{k}^{T} y_{k}\right)^{2},\left\|s_{k}\right\|^{4}\right\}} \cdot\left(y_{k} y_{k}^{T}\right) .
$$

Our $\lambda_{k}$ is guaranteed to be meaningful by $\max \left\{\left(s_{k}^{T} y_{k}\right)^{2},\left\|s_{k}\right\|^{4}\right\}>0$.

Hence, our stochastic LBFGS algorithm updates $B_{k}$ is

$$
B_{k}=B_{k-1}+\frac{y_{k-1}^{*} y_{k-1}^{* T}}{s_{k-1}^{T} s_{k-1}}-\frac{B_{k-1} s_{k-1} s_{k-1}^{T} B_{k-1}}{s_{k-1}^{T} B_{k-1} s_{k-1}} .
$$


Using the Sherman-Morrison-Woodbury formula, we can update $H_{k}=B_{k}^{-1}$ as

$$
H_{k}=\left(I-\frac{s_{k-1} y_{k-1}^{T}}{s_{k-1}^{T} y_{k-1}}\right) H_{k-1}\left(I-\frac{s_{k-1} y_{k-1}^{T}}{s_{k-1}^{T} y_{k-1}}\right)+\frac{s_{k-1} s_{k-1}^{T}}{s_{k-1}^{T} y_{k-1}} .
$$

Through simple observation, we can find the fact that, when the function is nonconvex, we can not guarantee that $s_{k}^{T} y_{k}^{*}>0$ is true. Thus, we add some additional settings to the algorithm to ensure the nonnegativity of $s_{k}^{T} y_{k}^{*}$. Define the index set $\mathcal{K}$ as follows:

$$
\mathcal{K}=\left\{i: s_{k}^{T} y_{k}^{*} \geq m\left\|s_{k}\right\|^{2}\right\}
$$

where $m$ is a positive constant.

Hence, our modified stochastic L-BFGS algorithm updates (18) and (19):

$$
\begin{aligned}
& B_{k}=\left\{\begin{array}{c}
B_{k-1}+\frac{y_{k-1}^{*} y_{k-1}^{* T}}{s_{k-1}^{T} s_{k-1}}-\frac{B_{k-1} s_{k-1} s_{k-1}^{T} B_{k-1}}{s_{k-1}^{T} B_{k-1} s_{k-1}}, \quad \text { if } \quad k \in K, \\
B_{k-1}, \quad \text { otherwise, }
\end{array}\right. \\
& H_{k}=\left\{\begin{array}{c}
H_{k}=\left(I-\frac{s_{k-1} y_{k-1}^{T}}{s_{k-1}^{T} y_{k-1}}\right) H_{k-1}\left(I-\frac{s_{k-1} y_{k-1}^{T}}{s_{k-1}^{T} y_{k-1}}\right)+\frac{s_{k-1} s_{k-1}^{T}}{s_{k-1}^{T} y_{k-1}}, \\
H_{k-1}, \quad \text { otherwise. }
\end{array}\right.
\end{aligned}
$$

As is known to all, the cost of calculating $H_{k}$ through (19) is very huge when $n$ is tremendously large. Hence, the LBFGS method is usually used instead of the BFGS method to overcome the poser of a large amount of calculation in large-scale optimization problems. The advantage of LBFGS is that it only uses curvature information and does not need to store the update matrix, which can effectively reduce the computational cost: Use (6) to iterate

$$
H_{k, i}=\left(I-\rho_{j} s_{j} y_{j}^{* T}\right) H_{k, i-1}\left(I-\rho_{j} y_{j}^{*} s_{j}^{T}\right)+\rho_{j} s_{j} s_{j}^{T}, \quad j=k-(r-i) ; i=0, \ldots, r-1,
$$

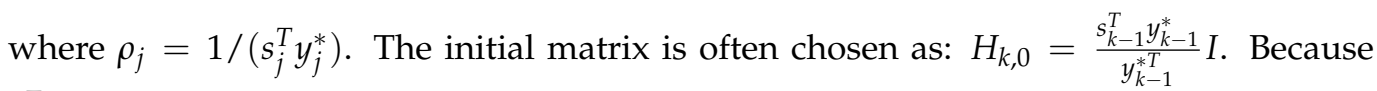
$s_{k-1}^{T} y_{k-1}^{*}$ may be exceedingly close to 0 , we set

$$
H_{k, 0}=\gamma_{k}^{-1} I
$$

and

$$
\gamma_{k}=\max \left\{\frac{\left\|y_{k-1}^{*}\right\|}{s_{k-1}^{T} y_{k-1}^{*}}, \delta\right\} \geq \delta
$$

where $\delta$ is a given constant.

Therefore, our modified stochastic L-BFGS algorithm is outlined in Algorithm 1.

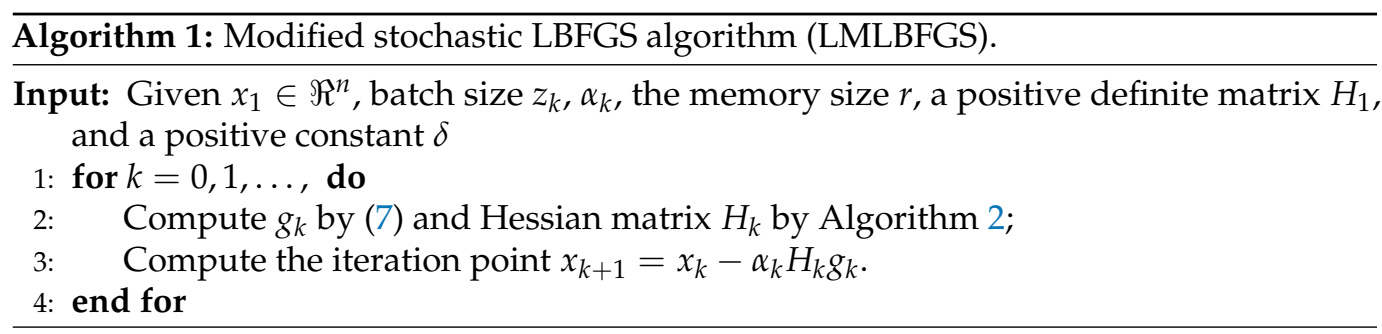

\subsection{Extension of Our LMLBFGS Algorithm with Variance Reduction}

Recently, using variance reduction technology in stochastic optimization methods can make the algorithm have better properties. Motivated by the development of the SVRG method for nonconvex problems, we present a new modified stochastic LBFGS algorithm 
(called LMLBFGS-VR) with a variance reduction technique for a faster convergence speed, as shown in Algorithm 3.

In LMLBFGS-VR, the mini-batch stochastic gradient is defined as

$$
g(x)=\frac{1}{|\mathscr{Z}|} \sum_{i \in \mathscr{Z}} \nabla f_{i}(x), \quad \mathscr{Z} \subset\{1,2, \ldots, n\} .
$$
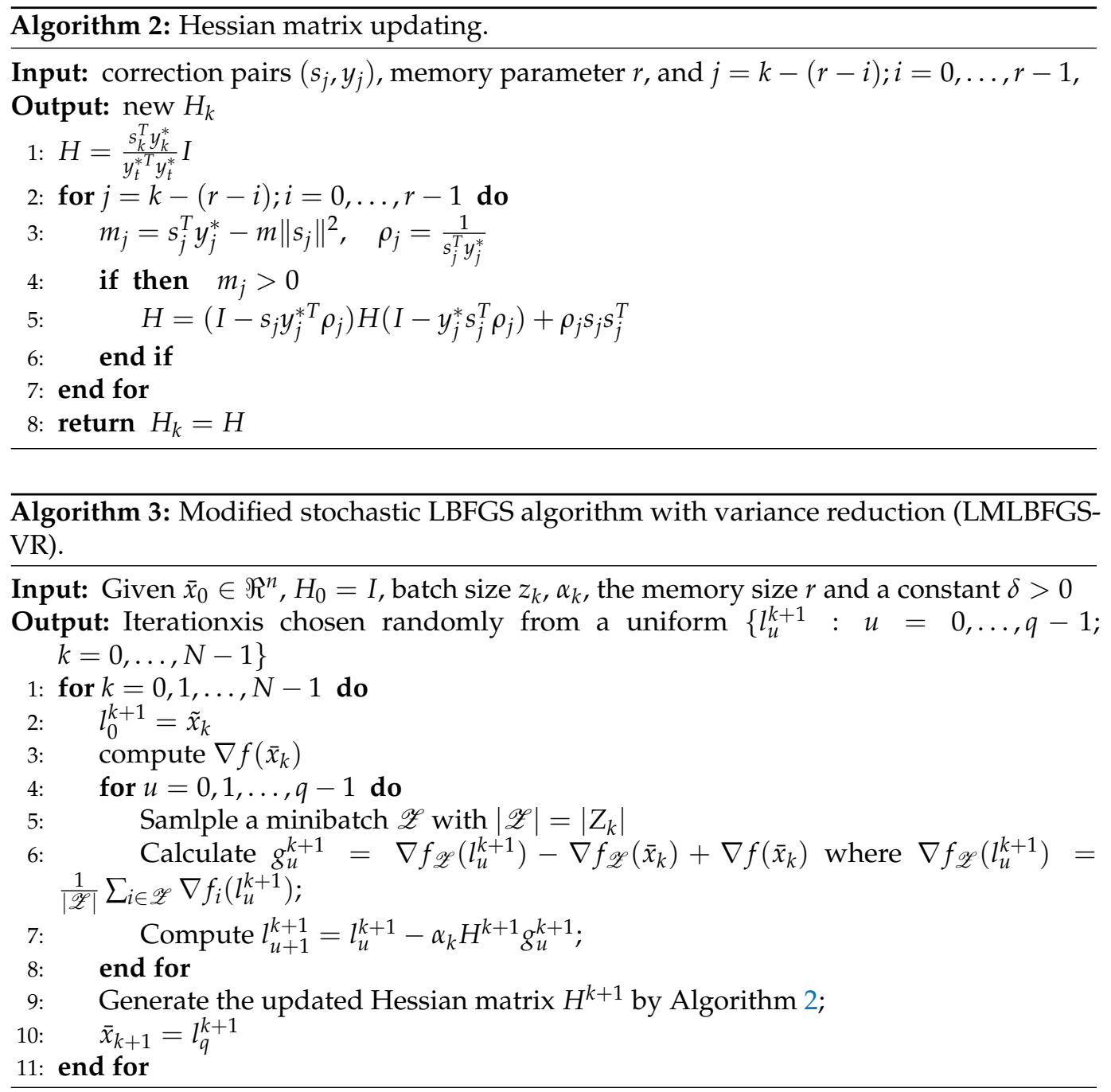

\section{Global Convergence Analysis}

In this section, the convergence of Algorithms 1 and 3 will be discussed and analyzed.

\subsection{Basic Assumptions}

In the algorithm, it is assumed that the step size satisfies

$$
\sum_{k=1}^{+\infty} \alpha_{k}=+\infty, \quad \sum_{k=1}^{+\infty} \alpha_{k}<+\infty
$$

Assumption 1. $f: \Re^{D_{x}} \rightarrow \Re$ is continuously differentiable and for any $x \in \Re^{n}, f(x)$ is bounded below. This means that there is constant $L>0$ that makes

$$
\left\|\nabla f\left(l_{1}\right)-\nabla f\left(l_{2}\right)\right\| \leq L\left\|l_{1}-l_{2}\right\|
$$

for any $l_{1}, l_{2} \in \Re^{n}$. 
Assumption 2. The noise level of the gradient estimation $\sigma$ such that

$$
\begin{gathered}
\mathbb{E}_{\tau_{k}}\left[g\left(x_{k}, \tau_{k}\right)\right]=\nabla f\left(x_{k}\right), \\
\mathbb{E}_{\tau_{k}}\left[\left\|g\left(x_{k}, \tau_{k}\right)-\nabla f\left(x_{k}\right)\right\|^{2}\right] \leq \sigma^{2},
\end{gathered}
$$

where $\sigma>0$ and $\mathbb{E}_{\tau_{k}}[\cdot]$ denotes the expectation taken with respect to $\tau_{k}$.

Assumption 3. There are positive $h_{1}$ and $h_{2}$ such that

$$
h_{1} I \preceq H_{k} \preceq h_{2} I .
$$

Our random variables $\tau_{k}$ are defined as follows: $\tau_{k}=\left(\tau_{k, 1}, \ldots, \tau_{k, z_{k}}\right)$ are the random samplings in the $k$-th iteration, and $\tau_{[k]}=\left(\tau_{1}, \ldots, \tau_{k}\right)$ are the random samplings in the first $k$-th iterations.

Assumption 4. For any $k \geq 2$, the random variable $H_{k}$ depends only on $\tau_{[k-1]}$.

From (2) and (4), we can get

$$
\mathbb{E}\left[H_{k} g_{k} \mid \tau_{[k]}\right]=H_{k} g_{k}
$$

3.2. Key Propositions, Lemmas, and Theorem

Lemma 1. If Assumptions $1-4$ hold and $\alpha_{k} \leq \frac{h_{1}}{L h_{2}^{2}}$ for all $k$, we have

$$
\mathbb{E}\left[f\left(x_{k+1}\right) \mid x_{k}\right] \leq-\frac{1}{2} \alpha_{k} h_{1}\left\|\nabla f\left(x_{k}\right)\right\|^{2}+f\left(x_{k}\right)+\frac{L \sigma^{2} h_{2}^{2}}{2 z_{k}} \alpha_{k^{\prime}}^{2}
$$

where the conditional expectation is taken with respect to $\tau_{k}$.

Proof.

$$
\begin{aligned}
f\left(x_{k+1}\right) \leq & f\left(x_{k}\right)+\left\langle\nabla f\left(x_{k}\right), x_{k+1}-x_{k}\right\rangle+\frac{L}{2}\left\|x_{k+1}-x_{k}\right\| \\
= & f\left(x_{k}\right)-\alpha_{k}\left\langle\nabla f\left(x_{k}\right), H_{k} g_{k}\right\rangle+\frac{L \alpha_{k}^{2}}{2}\left\|H_{k} g_{k}\right\|^{2} \\
\leq & f\left(x_{k}\right)-\alpha_{k}\left\langle\nabla f\left(x_{k}\right), H_{k} \nabla f\left(x_{k}\right)\right\rangle-\alpha_{k}\left\langle\nabla f\left(x_{k}\right), H_{k}\left(g_{k}-\nabla f\left(x_{k}\right)\right)\right\rangle \\
& +\frac{L \alpha_{k}^{2} h_{2}^{2}}{2}\left\|g_{k}\right\|^{2} .
\end{aligned}
$$

Taking expectation with respect to $\tau_{k}$ on both sides of (31) conditioned on $x_{k}$, we gain

$$
\mathbb{E}\left[f\left(x_{k+1}\right) \mid x_{k}\right] \leq f\left(x_{k}\right)-\alpha_{k}\left\langle\nabla f\left(x_{k}\right), H_{k} \nabla f\left(x_{k}\right)\right\rangle+\frac{L \alpha_{k}^{2} h_{2}^{2}}{2} \mathbb{E}\left[\left\|g_{k}\right\|^{2} \mid x_{k}\right],
$$

where we use the fact that $\mathbb{E}\left[\left(g_{k}-\nabla f\left(x_{k}\right)\right) \mid x_{k}\right]=0$. From Assumption 2, it follows that

$$
\begin{aligned}
\mathbb{E}\left[\left\|g_{k}\right\|^{2} \mid x_{k}\right] & =\mathbb{E}\left[\left\|g_{k}-\nabla f\left(x_{k}\right)+\nabla f\left(x_{k}\right)\right\|^{2} \mid x_{k}\right] \\
& =\mathbb{E}\left[\left\|\nabla f\left(x_{k}\right)\right\|^{2} \mid x_{k}\right]+2 \mathbb{E}\left[\left\|g_{k}-\nabla f\left(x_{k}\right)\right\| \mid x_{k}\right]+\mathbb{E}\left[\left\|g_{k}-\nabla f\left(x_{k}\right)\right\|^{2} \mid x_{k}\right] \\
& =\mathbb{E}\left[\left\|\nabla f\left(x_{k}\right)\right\|^{2} \mid x_{k}\right]+\mathbb{E}\left[\left\|g_{k}-\nabla f\left(x_{k}\right)\right\|^{2} \mid x_{k}\right] \\
& \leq\left\|\nabla f\left(x_{k}\right)\right\|^{2}+\frac{\sigma^{2}}{z_{k}} .
\end{aligned}
$$

Together with (32), we have 


$$
\mathbb{E}\left[f\left(x_{k+1}\right) \mid x_{k}\right] \leq f\left(x_{k}\right)-\left(\alpha_{k} h_{1}-\frac{L}{2} \alpha_{k}^{2} h_{2}^{2}\right)\left\|\nabla f\left(x_{k}\right)\right\|^{2}+\frac{L \sigma^{2} h_{2}^{2}}{2 z_{k}} \alpha_{k}^{2} .
$$

Then, combining that with $\alpha_{k} \leq \frac{h_{1}}{L h_{2}^{2}}$ implies (30).

Before proceeding further, the definition of supermartingale will be introduced [11].

Definition 1. Let $\left\{\mathscr{L}_{k}\right\}$ be an increasing sequence of $\sigma$-algebras. If $\left\{W_{k}\right\}$ is a stochastic process satisfying

(1) $C\left[\left|W_{k}\right|\right]<\infty$,

(2) $W_{k} \in \mathscr{L}_{k}$ and $\mathbb{E}\left[W_{k+1} \mid \mathscr{L}_{k}\right] \leq W_{k}$, for all $k$,

then $\left\{W_{k}\right\}$ is called a supermartingale.

Proposition 1. If $\left\{W_{k}\right\}$ is a nonnegative supermartingale, then $\lim _{k \rightarrow \infty} W_{k} \rightarrow W$ almost surely and $\mathbb{E}[W] \leq \mathbb{E}\left[W_{0}\right]$.

Lemma 2. Let $\left\{x_{k}\right\}$ be generated by Algorithm 1, where the batch size $z_{k}=z$, for all $k$. Then, there is a constant $M_{0}$ such that

$$
\mathbb{E}\left[f\left(x_{k}\right)\right] \leq M_{0}
$$

for all $k$.

Proof. For convenience of explanation, we have the following definitions:

$$
w_{k}=\frac{1}{2} \alpha_{k} h_{1}\left\|\nabla f\left(x_{k}\right)\right\|^{2}, \quad \psi_{k}=f\left(x_{k}\right)+\frac{L \sigma^{2} h_{2}^{2}}{2 z} \sum_{i=k}^{\infty} \alpha_{i}^{2} .
$$

Let $f$ be the the lower bound of the function and $W_{k}$ be the $\sigma$-algebra measuring $x_{k}$ $w_{k}$ and $\overline{\psi_{k}}$. From the definition, we obtain

$$
\begin{aligned}
\mathbb{E}\left[\psi_{k+1} \mid W_{k}\right] & =\mathbb{E}\left[f\left(x_{k+1}\right) \mid W_{k}\right]+\frac{L \sigma^{2} h_{2}^{2}}{2 z} \sum_{i=k+1}^{\infty} \alpha_{k}^{2} \\
& \leq f\left(x_{k}\right)-\frac{1}{2} \alpha_{k} h_{1}\left\|\nabla f\left(x_{k}\right)\right\|^{2}+\frac{L \sigma^{2} h_{2}}{2 z} \sum_{i=k+1}^{\infty} \alpha_{k}^{2} \\
& =\psi_{k}-w_{k} .
\end{aligned}
$$

Hence, we obtain

$$
\mathbb{E}\left[\psi_{k+1}-\underline{f} \mid W_{k}\right] \leq \psi_{k}-w_{k}-\underline{f}
$$

As a result, we have

$$
0 \leq \mathbb{E}\left[\psi_{k+1}-\underline{f}\right] \leq \psi_{1}-\underline{f}<\infty
$$

\subsection{Global Convergence Theorem}

In this part, we provide the convergence analysis of the proposed Algorithms 1 and 3.

Theorem 1. Assume that Assumptions 1-4 hold for $\left\{x_{k}\right\}$ generated by Algorithm 1, where the batch size is $z_{k}=z$. The step size satisfies (24) and $\alpha_{k} \leq \frac{h_{1}}{L h_{2}}$. Then, we have

$$
\lim _{k \rightarrow \infty} \inf \mathbb{E}\left[\left\|\nabla f\left(x_{k}\right)\right\|^{2}\right]=0 \quad \text { with probability } 1 \text {. }
$$


Proof. According to Definition $1, \psi_{k}-f$ is a supermartingale. Hence, there exists a $\psi$ such that $\lim _{k \rightarrow \infty}$ with probability 1 , and $\mathbb{E}[\psi] \leq \mathbb{E}\left[\psi_{1}\right]$ (Proposition 1). Form (36), we have $\mathbb{E}\left[w_{k}\right] \leq \mathbb{E}\left[\psi_{k}\right]-\mathbb{E}\left[\psi_{k+1}\right]$. Thus,

$$
\mathbb{E}\left[\sum_{k=1}^{\infty} w_{k}\right] \leq \sum_{k=1}^{\infty}\left(\mathbb{E}\left[\psi_{k}\right]-\mathbb{E}\left[\psi_{k+1}\right]\right) \leq \infty,
$$

which means that

$$
\sum_{k=1}^{\infty} w_{k}=\frac{h_{1}}{2} \sum \alpha_{k}\left\|\nabla f\left(x_{k}\right)\right\|^{2}<+\infty \quad \text { with probability } 1 .
$$

Since (24), it follows that (48) holds.

Next, the convergence of the algorithm can be given.

Theorem 2. If Assumptions 1, 2, and 4 hold for $\left\{x_{k}\right\}$ generated by Algorithm 1, where the batch size is $z_{k}=z$. The step size satisfies (24) and $\alpha_{k} \leq \frac{h_{1}}{L h_{2}}$. Then, we have

$$
\lim _{k \rightarrow \infty} \inf \mathbb{E}\left[\left\|\nabla f\left(x_{k}\right)\right\|^{2}\right]=0 \quad \text { with probability } 1 .
$$

Proof. The proof will be established by contradiction, and the discussion is listed as follows. According to the definition of $y_{k}^{*}$, we have

$$
\begin{aligned}
s_{j}^{T} y_{j}^{*} & =s_{j}^{T} y_{j}+\frac{2\left[f\left(x_{j-1}\right)-f\left(x_{j}\right)\right]+\left(g_{j}+g_{j-1}\right)^{T} s_{j}}{\max \left\{\left(s_{j}^{T} y_{j}\right)^{2},\left\|s_{j}\right\|^{4}\right\}} \cdot\left(s_{j}^{T} y_{j} y_{j}^{T} s_{j}\right) \\
& \leq s_{j}^{T} y_{j}+\frac{2\left[f\left(x_{j-1}\right)-f\left(x_{j}\right)\right]+\left(g_{j}+g_{j-1}\right)^{T} s_{j}}{\left(s_{j}^{T} y_{j}\right)^{2}} \cdot\left(s_{j}^{T} y_{j} y_{j}^{T} s_{j}\right) \\
& =s_{j}^{T} y_{j}+2\left[f\left(x_{j-1}\right)-f\left(x_{j}\right)\right]+\left(g_{j}+g_{j-1}\right)^{T} s_{j} \\
& =s_{j}^{T} y_{j}-2 g\left(x_{j-1}+\theta\left(x_{j}-x_{j-1}\right)\right)^{T} s_{j}+\left(g_{j}+g_{j-1}\right)^{T} s_{j} \\
& =2 s_{j}^{T}\left(g_{j}-g\left(x_{j-1}+\theta\left(x_{j}-x_{j-1}\right)\right)\right) \\
& \leq 2(1-\theta) L\left\|s_{j}\right\|\left\|\left(x_{j}-x_{j-1}\right)\right\| \\
& =2(1-\theta) L\left\|s_{j}\right\|^{2},
\end{aligned}
$$

where $\theta \in(0,1)$. It is easy to see that

$$
m\left\|s_{j}\right\|^{2} \leq s_{j}^{T} y_{j}^{*} \leq \Lambda\left\|s_{j}\right\|^{2},
$$

where $\Lambda$ is a positive constant.

According to the definition of $y_{k}^{*}$, we have 


$$
\begin{aligned}
\left\|y_{j}^{*}\right\|= & \left\|y_{j}+\frac{2\left[f\left(x_{j-1}\right)-f\left(x_{j}\right)\right]+\left(g_{j}+g_{j-1}\right)^{T} s_{j}}{\max \left\{\left(s_{j}^{T} y_{j}\right)^{2},\left\|s_{j}\right\|^{4}\right\}} \cdot\left(y_{j} y_{j}^{T}\right) \cdot s_{j}\right\| \\
\leq & \left\|y_{j}\right\|+\left|2\left[f\left(x_{j-1}\right)-f\left(x_{j}\right)\right]+\left(g_{j}+g_{j-1}\right)^{T} s_{j}\right|\left\|\frac{y_{j} y_{j}^{T}}{\left\|s_{j}\right\|^{4}} s_{j}\right\| \\
\leq & \left\|y_{j}\right\|+\left|-2 g\left(x_{j-1}+\theta\left(x_{j}-x_{j-1}\right)\right)^{T} s_{j}+\left(g_{j}+g_{j-1}\right)^{T} s_{j}\right| \cdot \frac{\left\|y_{j} y_{j}^{T}\right\|}{\left\|s_{j}\right\|^{4}}\left\|s_{j}\right\| \\
\leq & \left\|y_{j}\right\|+\mid\left(g_{j}-g\left(x_{j-1}+\theta\left(x_{j}-x_{j-1}\right)\right)^{T} s_{j}\right. \\
& +\left(g_{j-1}-g\left(x_{j-1}+\theta\left(x_{j}-x_{j-1}\right)\right)^{T} s_{j}\left(g_{j}+g_{j-1}\right)^{T} s_{j} \mid \cdot \frac{\left\|y_{j} y_{j}^{T}\right\|}{\left\|s_{j}\right\|^{3}}\right. \\
\leq & L\left\|s_{j}\right\|+\left(L(1-\theta)\left\|s_{j}\right\|^{2}+L \theta\left\|s_{j}\right\|^{2}\right) \cdot \frac{\left\|y_{j}\right\|\left\|y_{j}\right\|}{\left\|s_{j}\right\|^{3}} \\
= & 2 L\left\|s_{j}\right\| .
\end{aligned}
$$

From (41) and (42), we have

$$
\lambda \leq \frac{\left\|y_{j}^{*}\right\|^{2}}{s_{j}^{T} y_{j}^{*}} \leq \frac{(2 L)^{2}\left\|s_{j}\right\|^{2}}{\lambda\left\|s_{j}\right\|^{2}}=\frac{(2 L)^{2}}{\lambda}=M_{0},
$$

where the first inequality is derived from the quasi Newton condition. This equation shows that the eigenvalue of our initial matrix $B_{k}^{(0)}=\frac{y_{k}^{* T} y_{k}^{*}}{s_{k}^{T} y_{k}^{*}} I$ is bounded, and the eigenvalue is much greater than 0 .

Instead of directly analyzing the properties of $H_{k}$, we get the results by analyzing the properties of $B_{k}$. In this situation, the limited memory quasi-Newton updating formula is as follows:

(i) $B_{k}^{(0)}=\frac{y_{k}^{* T} y_{k}^{*}}{s_{k}^{T} y_{k}^{*}} I$.

(ii) for $i=0, \ldots, r-1, j=k-(r-i)$ and

$$
B_{k}^{(i+1)}=B_{k}^{(i)}-\frac{B_{k}^{(i)} s_{j} s_{j}^{T} B_{k}^{(i)}}{s_{j}^{T} B_{k}^{(i)} s_{j}}+\frac{y_{j}^{*} y_{j}^{* T}}{s_{j}^{T} y_{j}^{*}} .
$$

The trace of matrix $B$ is defined as $\operatorname{tr}(B)$. Then, from (43) and (44), and the boundedness of $\left\{\left\|B_{k}^{(0)}\right\|\right\}$, we obtain

$$
\begin{aligned}
\operatorname{tr}\left(B_{k+1}\right) & \leq \operatorname{tr}\left(B_{k}^{(0)}\right)+\sum_{i=1}^{r} \frac{\left\|y_{j}^{*}\right\|^{2}}{s_{j}^{T} y_{j}^{*}} \\
& \leq \operatorname{tr}\left(B_{k}^{(0)}\right)+r \Lambda \\
& =M_{1} .
\end{aligned}
$$

The determinant of $B_{k}$ is now considered because the determinant can be used to prove that the minimum eigenvalue of matrix $B$ is uniformly bounded. From the theory in [12], we can get the following equation about matrix determinant: 


$$
\begin{aligned}
\operatorname{det}\left(B_{k+1}\right) & =\operatorname{det}\left(B_{k}^{(0)}\right) \prod_{i=1}^{r} \frac{y_{j_{i}}^{* T} s_{j_{i}}}{s_{j_{i}}^{T} B_{k}^{(i-1)} s_{j_{i}}} \\
& =\operatorname{det}\left(B_{k}^{(0)}\right) \prod_{i=1}^{r} \frac{y_{j_{i}}^{* T} s_{j_{i}}}{s_{j_{i}}^{T} s_{j_{i}}} \frac{s_{j_{i}}^{T} s_{j_{i}}}{s_{j_{i}}^{T} B_{k}^{(i-1)} s_{j_{i}}} .
\end{aligned}
$$

It can be obtained from (45) that the maximum eigenvalue of matrix $B_{j}$ is uniformly bounded. Therefore, according to (41) and combining the fact that the smallest eigenvalue of $B_{k}^{(0)}$ is bounded away from zero, the following equation is obtained:

$$
\begin{aligned}
\operatorname{det}\left(B_{k+1}\right) & \geq \operatorname{det}\left(B_{k}^{(0)}\right)\left(\frac{\lambda}{M_{1}}\right)^{r} \\
& \geq M_{2} .
\end{aligned}
$$

In this way, the maximum eigenvalue and the minimum eigenvalue of matrix $B_{j}$ are uniformly bounded and much greater than 0 . Therefore, we can get

$$
h_{1} I \preceq H_{k} \preceq h_{2} I \text {, }
$$

where $h_{1}$ and $h_{2}$ are positive constants. According to Theorem 1 that we proved above, the convergence of our proposed Algorithm 1 can be obtained.

Corollary 1. If Assumptions 1, 2, and 4 hold for $\left\{x_{k}\right\}$ generated by Algorithm 3, where the batch size is $z_{k}=z$ and the step size satisfies (24) and $\alpha_{k} \leq \frac{h_{1}}{L h_{2}}$, then, we have

$$
\lim _{k \rightarrow \infty} \inf \mathbb{E}\left[\left\|\nabla f\left(x_{k}\right)\right\|^{2}\right]=0 \quad \text { with probability } 1 .
$$

\section{The Complexity of the Proposed Algorithm}

The convergence results of the algorithm have been discussed. Now, let us analyze the complexity of Algorithms 1 and 3.

Assumption 5. For any $k$, we have

$$
\alpha_{k}=\frac{h_{1}}{L h_{2}^{2}} k^{-\beta}, \quad \beta \in(0.5,1)
$$

Theorem 3. Suppose Assumptions 1-5 hold, $\left\{t_{k}\right\}$ is generated by Algorithm 1, and batch size $z_{k}=z$ for all $k$. Then, we have

$$
\frac{1}{N} \sum_{k=1}^{N} \mathbb{E}\left[\left\|\nabla f\left(t_{k}\right)\right\|^{2}\right] \leq \frac{2 L\left(M_{0}-\underline{f}\right) h_{2}^{2}}{h_{1}^{2}} N^{\beta-1}+\frac{\sigma^{2}}{(1-\beta) z}\left(N^{-\beta}-N^{-1}+\frac{1-\beta}{N}\right),
$$

where $N$ denotes the iteration number.

Moreover, for a given $\epsilon \in(0,1)$, to guarantee that

$$
\frac{1}{N} \sum_{k=1}^{N} \mathbb{E}\left[\left\|\nabla f\left(t_{k}\right)\right\|^{2}\right]<\epsilon
$$

the number of iterations $N$ needed is at most $O\left(\epsilon^{-\frac{1}{1-\epsilon}}\right)$.

Proof. Obviously, (49) satisfies (24) and the condition $\alpha_{k} \leq \frac{h_{1}}{L h_{2}^{2}}$. Then, taking expectations on both sides of (30) and summing over all $k$ yield 


$$
\begin{aligned}
\frac{1}{2} h_{1} \sum_{k=1}^{N} \mathbb{E}\left[\left\|\nabla f\left(t_{k}\right)\right\|^{2}\right] & \leq \sum_{k=1}^{N} \frac{1}{\alpha_{k}}\left(\mathbb{E}\left[f\left(t_{k}\right)\right]-\mathbb{E} f\left(t_{k+1}\right)\right)+\frac{L \sigma^{2} h_{2}^{2}}{2 z} \sum_{k=1}^{N} \alpha_{k} \\
& =\frac{1}{\alpha_{1}} f\left(t_{1}\right)+\sum_{k=2}^{N}\left(\frac{1}{\alpha_{k}}-\frac{1}{\alpha_{k-1}}\right) \mathbb{E}\left[f\left(t_{k}\right)\right]-\frac{\mathbb{E}\left[f\left(x_{N+1}\right)\right]}{\alpha_{N}}+\frac{L \sigma^{2} h_{2}^{2}}{2 z} \sum_{k=1}^{N} \alpha_{k} \\
& \leq \frac{M_{0}}{\alpha_{1}}+M_{0} \sum_{k=2}^{N}\left(\frac{1}{\alpha_{k}-\alpha_{k-1}}\right)-\frac{f}{\alpha_{N}}+\frac{L \sigma^{2} h_{2}^{2}}{2 z} \sum_{k=1}^{N} \alpha_{k} \\
& =\frac{M_{0}-\underline{f}}{\alpha_{N}}+\frac{L \sigma^{2} h_{2}^{2}}{2 z} \sum_{k=1}^{N} \alpha_{k} \\
& \leq \frac{L\left(M_{f}-\underline{f} h_{2}^{2}\right)}{h_{1}} N^{\beta}+\frac{\sigma^{2} h_{1}}{2(1-\beta) z}\left(N^{1-\beta}-\beta\right),
\end{aligned}
$$

which results in (50), where the second inequality is due to Lemma 2, and the last inequality is due to Theorem 1 .

Next, for a given $\epsilon>0$, in order to obtain $\frac{1}{N} \sum_{k=1}^{N} \mathbb{E}\left[\left\|\nabla f\left(x_{k}\right)\right\|^{2}\right] \leq \epsilon$, we only need the following equation:

$$
\frac{2\left(M_{0}-\underline{f}\right) L h_{2}^{2}}{h_{1}^{2}} N^{\beta-1}-\frac{\sigma^{2}}{(1-\beta) z}\left(N^{-1}-N^{-\beta}-\frac{1-\beta}{N}\right)<\epsilon .
$$

Since $\beta \in(0.5,1)$, it follows that the number of iterations $N$ needed is at most $O\left(\epsilon^{-\frac{1}{1-\beta}}\right)$.

Corollary 2. Assume that Assumptions 1, 3, 4 and (27) hold for $x_{k}$ generated by Algorithm 3 with batch size $z_{k}=z$ for all $k$. We also assume that $\alpha_{k}$ is specifically chosen as

$$
\alpha_{k}=\frac{h_{1}}{L h_{2}^{2}} k^{-\beta}
$$

with $\beta \in(0.5,1)$. Then,

$$
\frac{1}{N} \sum_{k=1}^{N} \mathbb{E}\left[\left\|\nabla f\left(x_{k}\right)\right\|^{2}\right] \leq \frac{2 L\left(M_{0}-\underline{f}\right) h_{2}^{2}}{h_{1}^{2}} N^{\beta-1}+\frac{\sigma^{2}}{(1-\beta) z}\left(N^{-\beta}-N^{-1}+\frac{1-\beta}{N}\right),
$$

where $N$ denotes the iteration number. Moreover, for a given $\epsilon \in(0,1)$, to guarantee that $\frac{1}{N} \sum_{k=1}^{N} \mathbb{E}\left[\left\|\nabla f\left(x_{k}\right)\right\|^{2}\right]<\epsilon$, the number of iterations $N$ needed is at most $O\left(\epsilon^{-\frac{1}{1-\epsilon}}\right)$.

\section{Numerical Results}

In this section, we focus on the numerical performances of the proposed Algorithm 3 for solving nonconvex empirical risk minimization (ERM) problems and nonconvex support vector machine (SVM) problems.

\subsection{Experiments with Synthetic Datasets}

The models of the nonconvex SVM problems and nonconvex ERM problems are given as follows: $\lambda>0$ is a regularization parameter.

Problem 1. The ERM problem with a nonconvex sigmoid loss function $[13,14]$ is formulated as follows:

$$
\min _{x \in \Re^{D x}} \frac{1}{n} \sum_{i=1}^{n} f_{i}(x)+\frac{\lambda}{2}\|x\|_{2}^{2}, \quad f_{i}(x)=\frac{1}{1+\exp \left(b_{i} a_{i}^{T} x\right)},
$$

where $a_{i} \in \Re^{d}$ and $b_{i} \in\{-1,1\}$ represent the feature vector and corresponding label, respectively. 
Problem 2. The nonconvex support vector machine (SVM) problem with a sigmoid loss function $[15,16]$ is formulated as follows:

$$
\min _{x \in \Re^{D x}} \frac{1}{n} \sum_{i=1}^{n} f_{i}(x)+\lambda\|x\|^{2}, \quad f_{i}(x)=1-\tanh \left(b_{i}\left\langle x, a_{i}\right\rangle\right) .
$$

We compare the proposed LMLBFGS-VR algorithm with SGD [1], SVRG [17] and SAGA [18], where the LMLBFGS-VR algorithms use a descent step size and other algorithms use a constant step size $\alpha_{k}$. The data sets in our experiments including Adult, IJCNN, Mnist, and Coctype. All the codes are written in MATLAB 2018b on a PC with AMD Ryzen $75800 \mathrm{H}$ with Radeon Graphics $3.20 \mathrm{GHz}$ and 16 GB of memory.

\subsection{Numerical Results for Problem 1}

In this subsection, we present the numerical results of LMLBFGS-VR, SGD, SVRG, and SAGA for solving Problem 1 on the four data sets. For LMLBFGS-VR algorithms, the step size is $\alpha_{k}=0.02 \times k^{-0.6}$, and the memory size is $r=10$ and $m=1 \times 10^{-5}$. The step size of other algorithms is chosen as 0.02 . The number of inner loop $q$ we chose as $n / V$ uniformly, where $V$ is the batch size. The batch-size is set to 100 for Adult, IJCNN, and Covtype, and for Mnist. In order to further test the performance of the algorithm, the regularization parameter is set to $10^{-3}, 10^{-4}$, or $10^{-5}$. The following pictures demonstrate the performance of different algorithms. Figures 1-4 show the convergence performance of all the stochastic algorithms for solving Problem 1 with $\lambda=1 \times 10^{-3}, \lambda=1 \times 10^{-4}$ or $\lambda=1 \times 10^{-5}$ on four different data sets. From Figures 1-4, we obtain that all the algorithms can solve the problem successfully. However, the proposed LMLBFGS- VR algorithms have significantly faster convergence speed than other algorithms. It is clear that the proposed algorithms, especially LMLBFGS-VR, have a great advantage for solving nonconvex support vector machine problems.
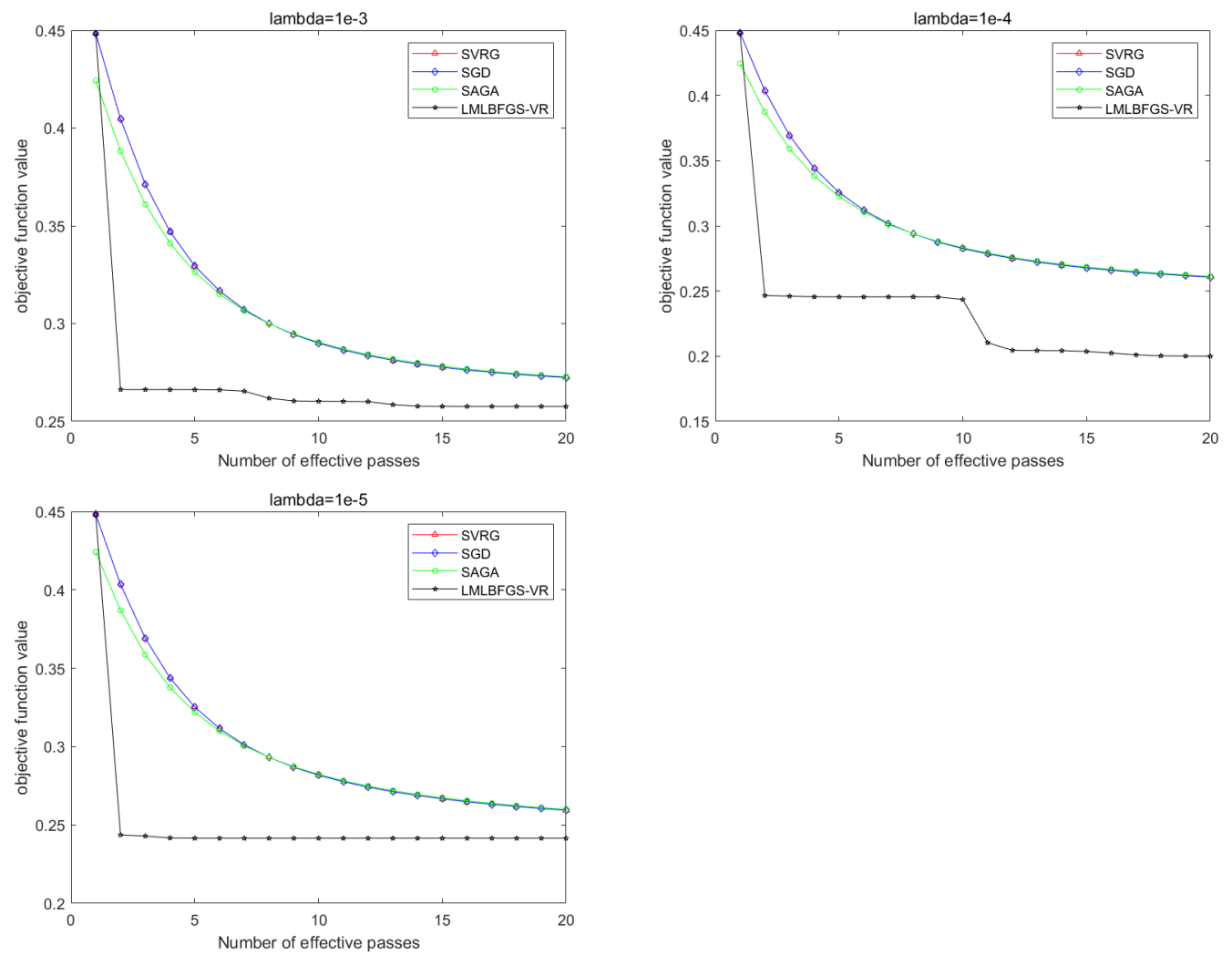

Figure 1. Comparison of all the algorithms for solving Problem 1 on Adult. From left to right: $\lambda=1 \times 10^{-3}, \lambda=1 \times 10^{-4}, \lambda=1 \times 10^{-5}$. 

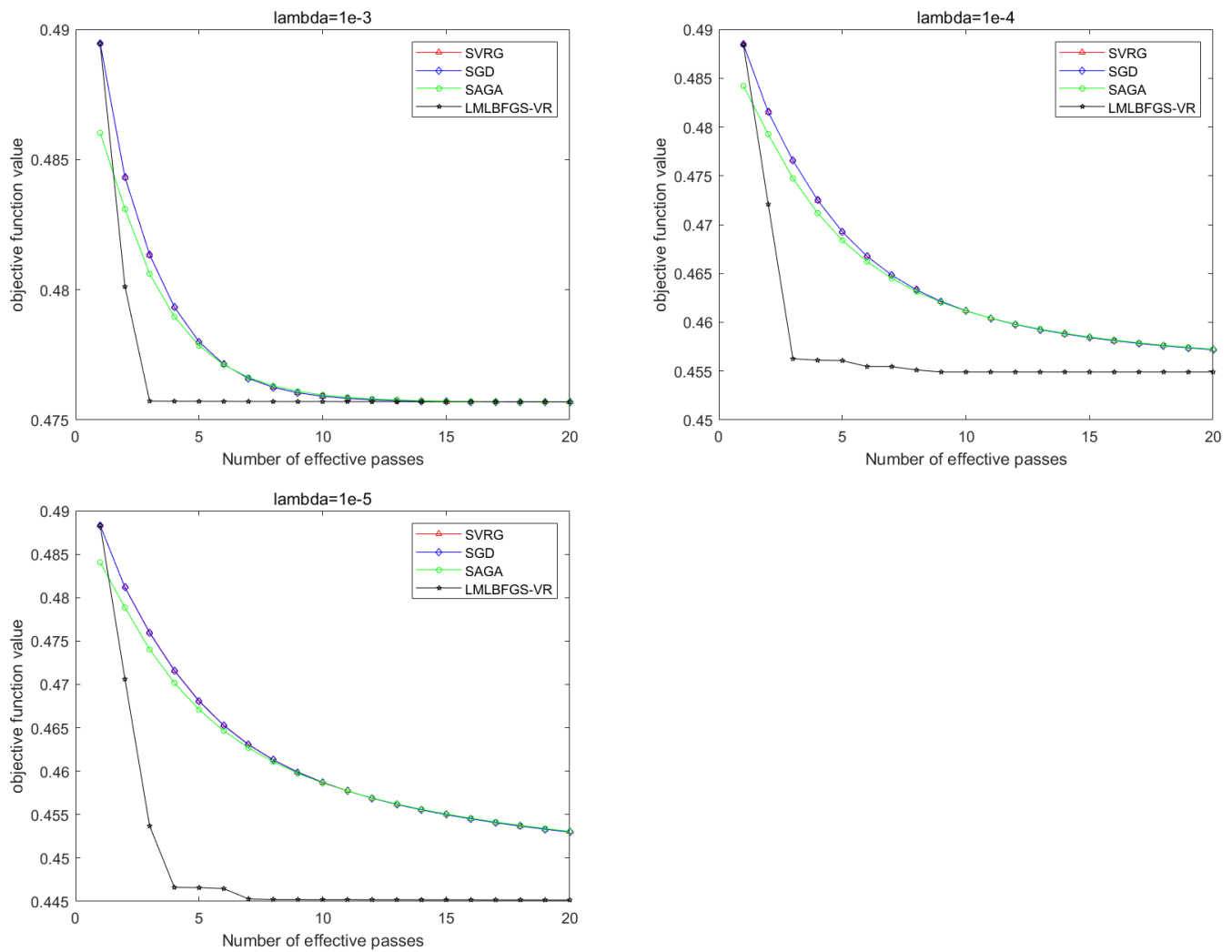

Figure 2. Comparison of all the algorithms for solving Problem 1 on Covtype. From left to right: $\lambda=1 \times 10^{-3}, \lambda=1 \times 10^{-4}, \lambda=1 \times 10^{-5}$.
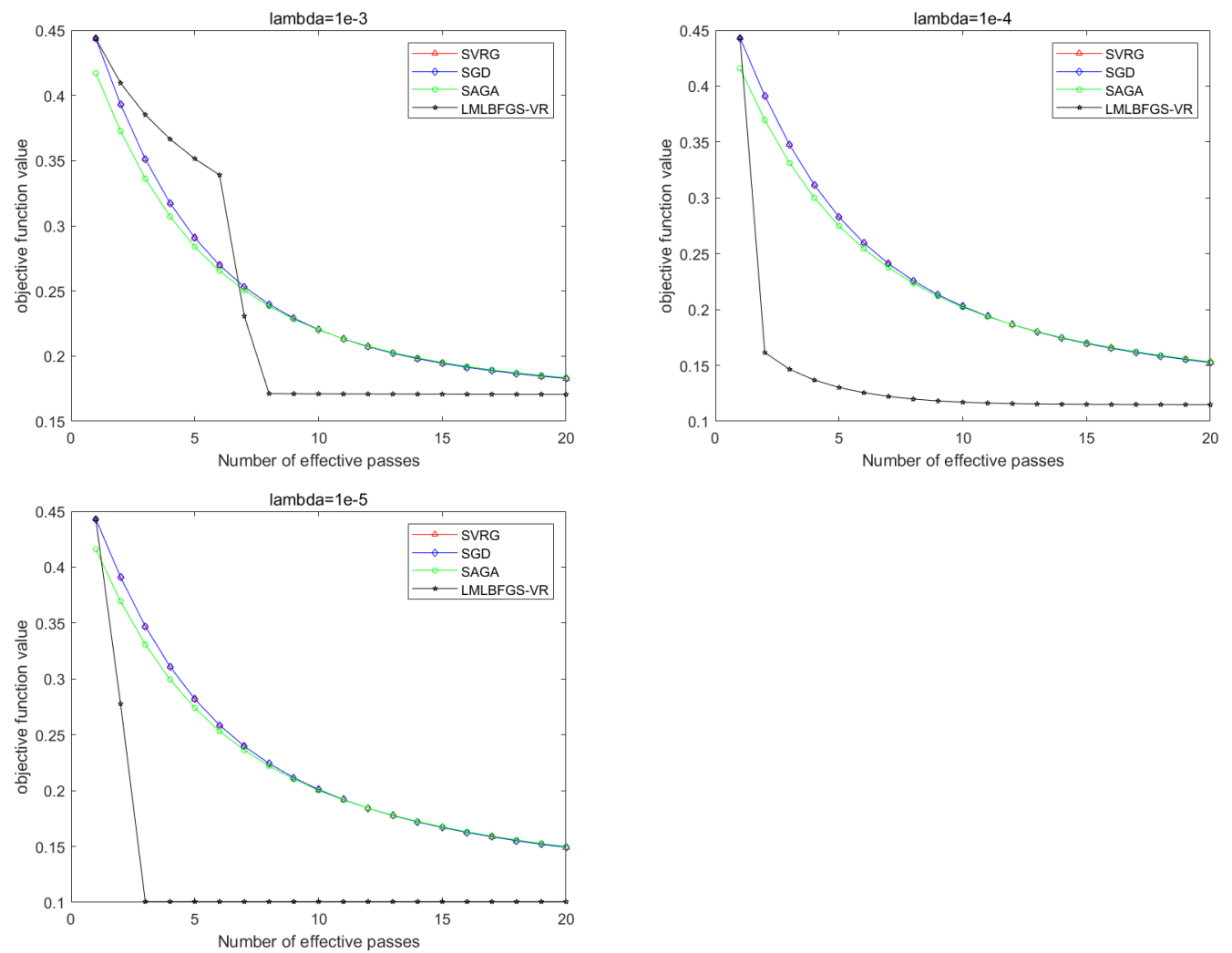

Figure 3. Comparison of all the algorithms for solving Problem 1 on IJCNN. From left to right: $\lambda=1 \times 10^{-3}, \lambda=1 \times 10^{-4}, \lambda=1 \times 10^{-5}$. 

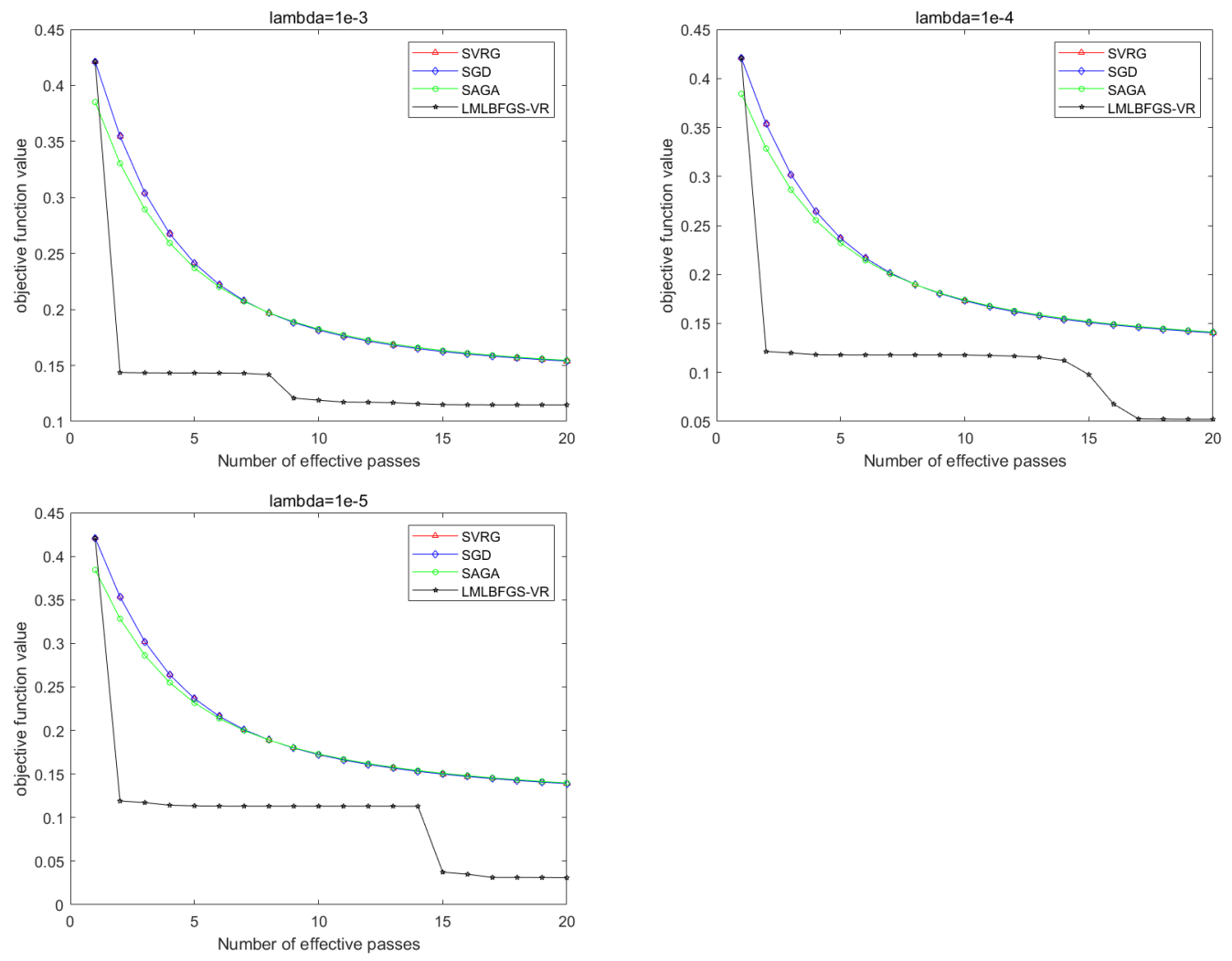

Figure 4. Comparison of all the algorithms for solving Problem 1 on mnist. From left to right: $\lambda=1 \times 10^{-3}, \lambda=1 \times 10^{-4}, \lambda=1 \times 10^{-5}$.

\subsection{Numerical Results for Problem 2}

The numerical results of LMLBFGS-VR, SGD, SVRG, and SAGA for solving Problem 2 on the four data sets are presented in this subsection. All parameters are the same as the above subsection, and the regularization parameter is also set to $1 \times 10^{-3}, 1 \times 10^{-4}$, or $1 \times 10^{-5}$. The following figures demonstrate the performance of all the stochastic algorithms. The $y$-axis is the objective function value, and the $x$-axis denotes the number of effective passes, where computing a full gradient or evaluatingncomponent gradients is regarded as an effective pass. Figures $4-8$ demonstrate that the convergence performance of our LMLBFGS-VR algorithms on the four data sets, which show that they remarkably outperform the other algorithms. When $\lambda=1 \times 10^{-3}$, the objective function is almost minimized by two effective passes. In contrast, the SGD, SVRG, and SAGA algorithms converge slightly slowly, where these algorithms only use first-order information. Due to the use of second-order information and limited memory technique, LMLBFGS-VR requires only a few effective passes to quickly minimize the function value. From Figures $4-8$, we find that, as $\lambda$ decreases, the value of the function decreases to a smaller value. Thus, we can choose a smaller $\lambda$ for practical problems. Combined with the previous discussion, our LMLBFGS-VR algorithms make great progress in improving the computing efficiency for nonconvex machine learning problems. 

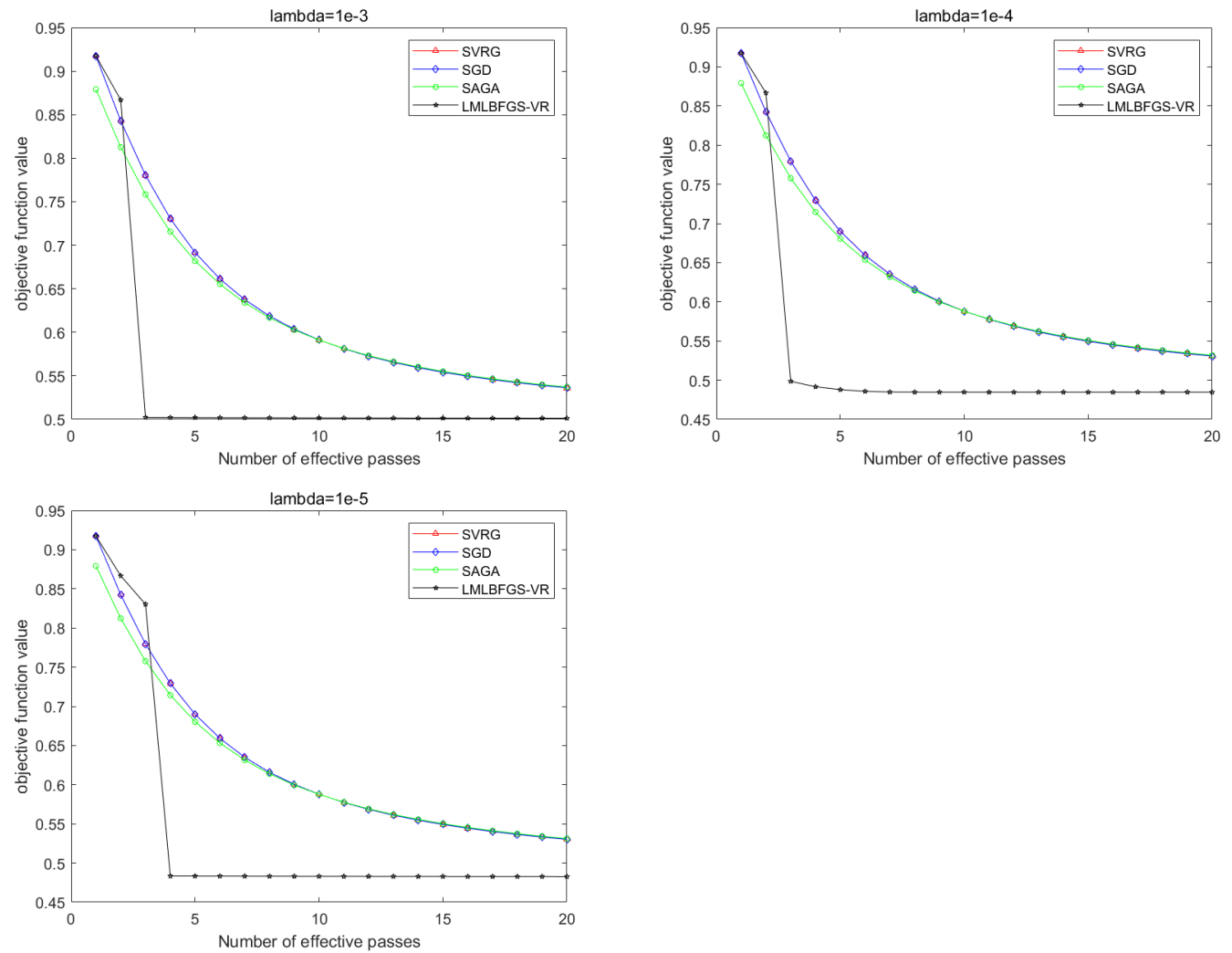

Figure 5. Comparison of all the algorithms for solving Problem 2 on Adult. From left to right: $\lambda=1 \times 10^{-3}, \lambda=1 \times 10^{-4}, \lambda=1 \times 10^{-5}$.
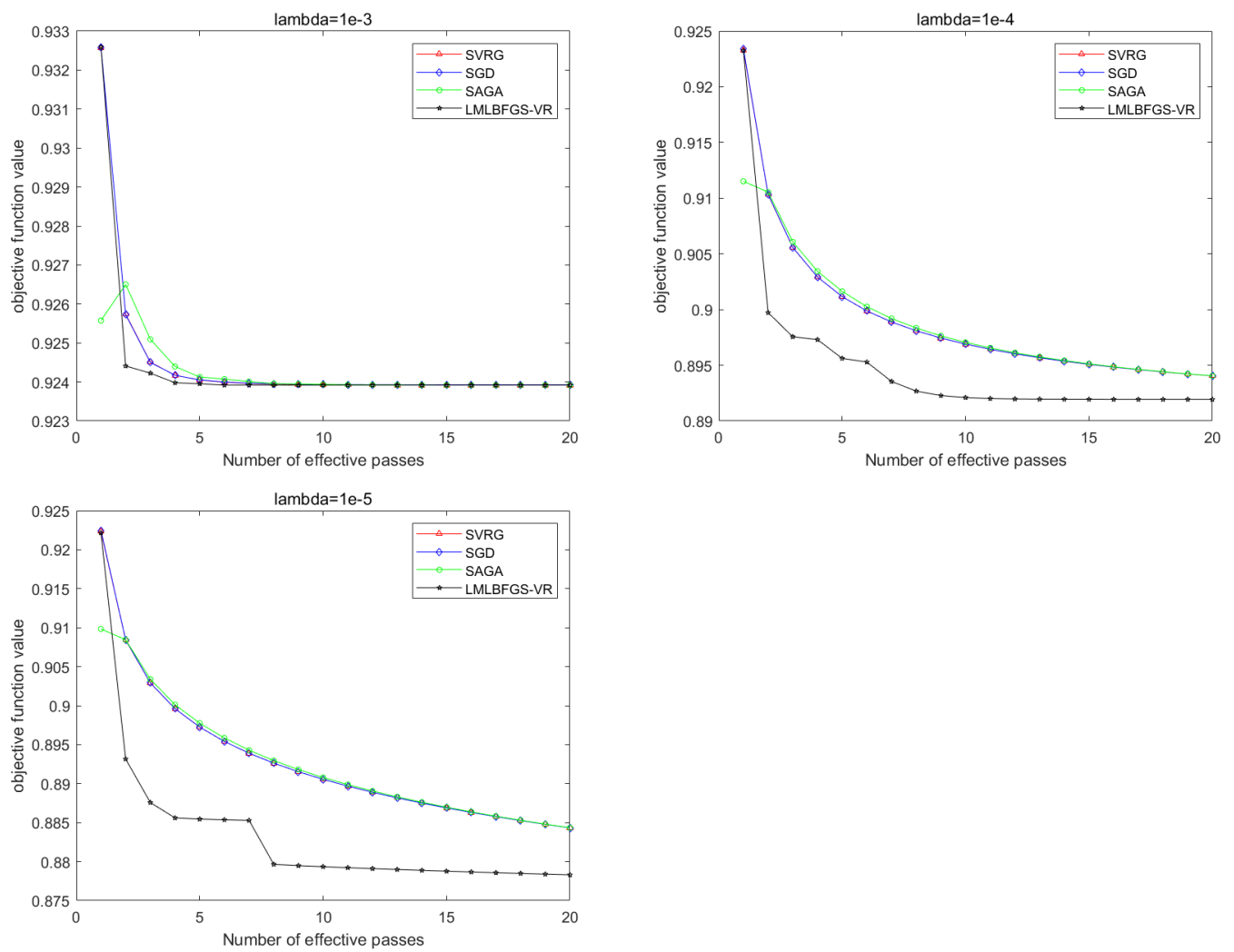

Figure 6. Comparison of all the algorithms for solving Problem 2 on Covtype. From left to right: $\lambda=1 \times 10^{-3}, \lambda=1 \times 10^{-4}, \lambda=1 \times 10^{-5}$. 

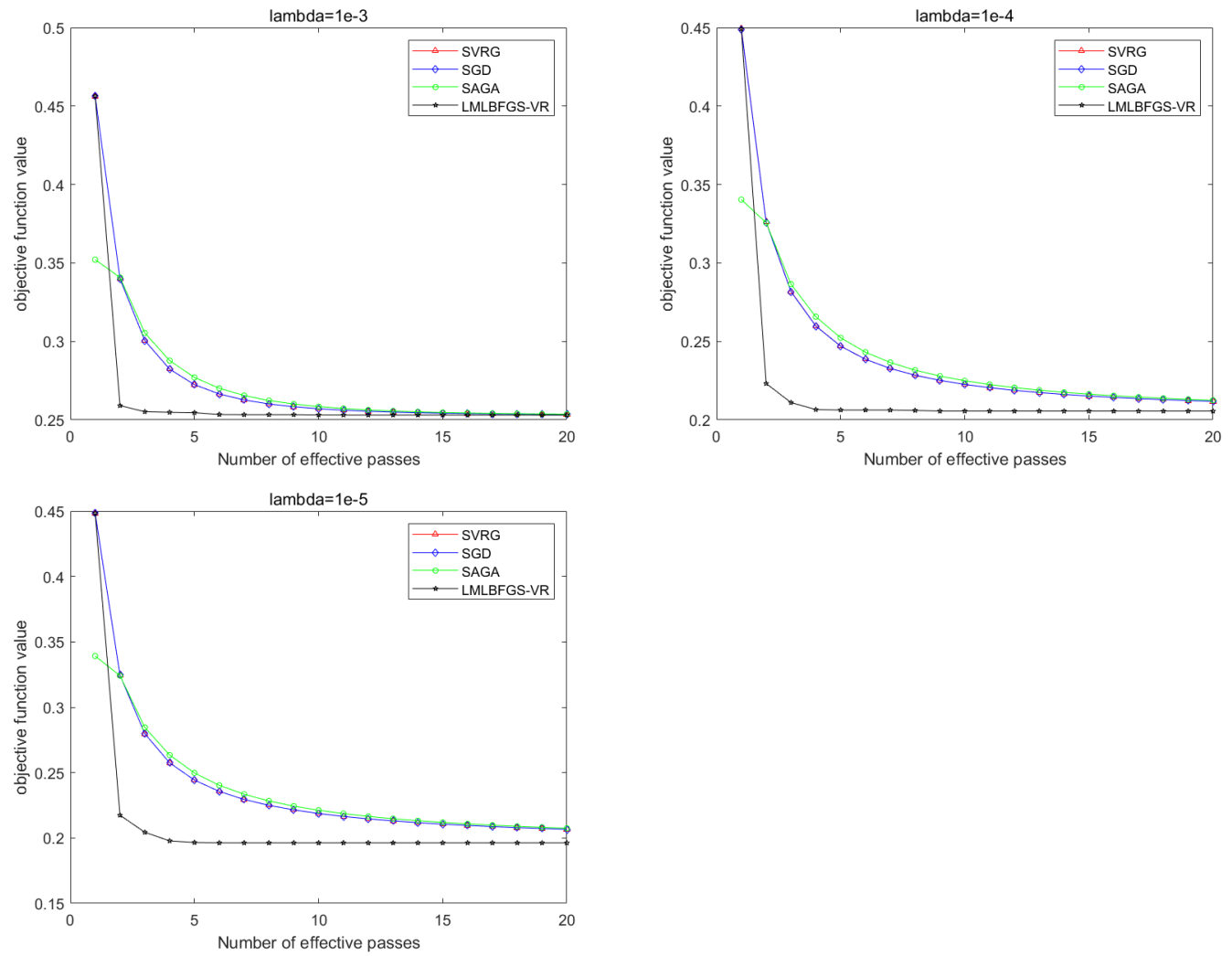

Figure 7. Comparison of all the algorithms for solving Problem 2 on IJCNN. From left to right: $\lambda=1 \times 10^{-3}, \lambda=1 \times 10^{-4}, \lambda=1 \times 10^{-5}$.
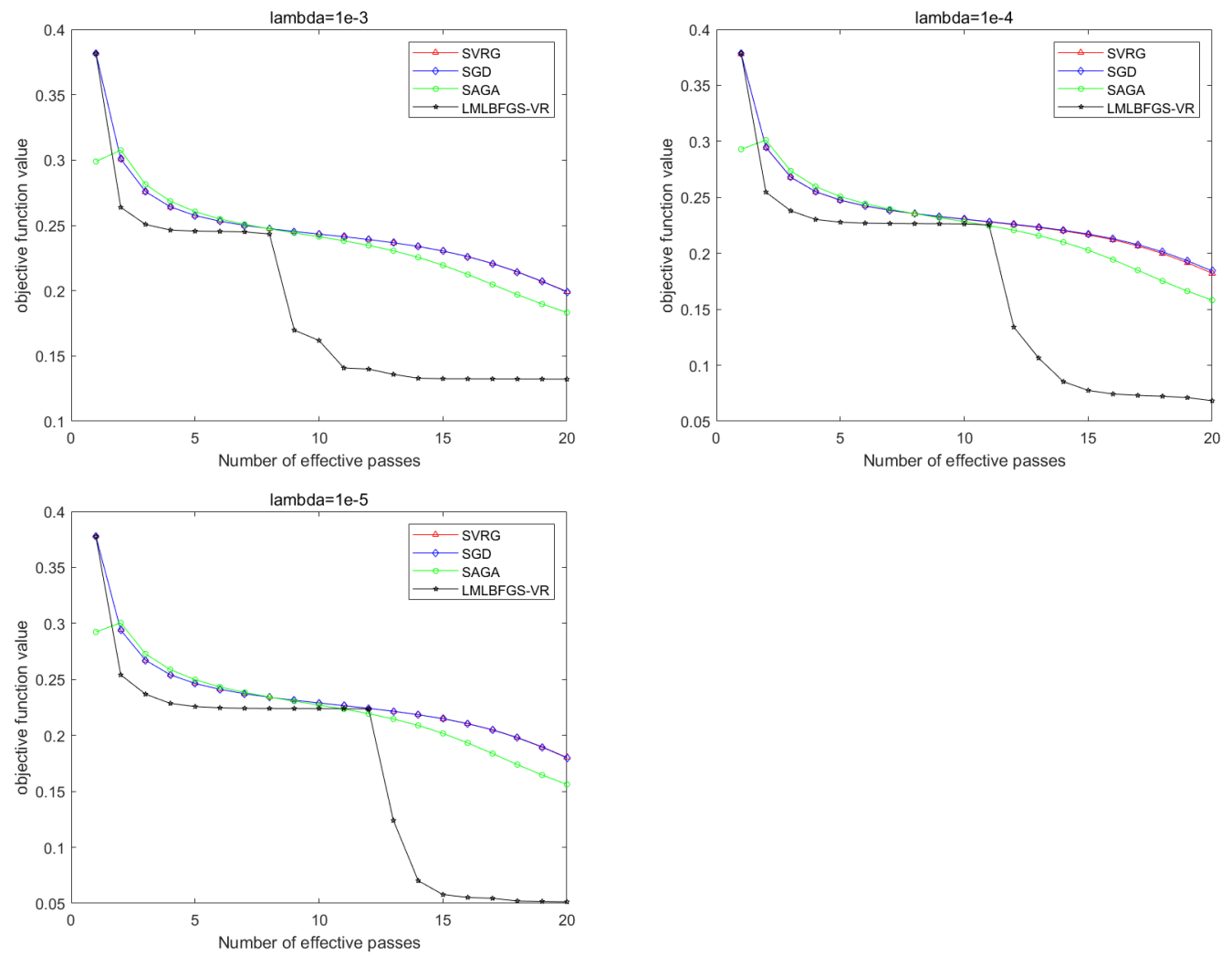

Figure 8. Comparison of all the algorithms for solving Problem 2 on mnist. From left to right: $\lambda=1 \times 10^{-3}, \lambda=1 \times 10^{-4}, \lambda=1 \times 10^{-5}$. 


\section{Conclusions}

In this paper, we proposed one efficient modified stochastic limited BFGS algorithms for solving nonconvex stochastic optimization. The proposed algorithms can preserve the positive definiteness of $H k$ without any convexity properties. The LMLBFGS-VR method with variance reduction was also presented to solve nonconvex stochastic optimization problems. Numerical experiments on nonconvex SVM problems and nonconvex ERM problems were performed to demonstrate the performance of the proposed algorithms, and the results indicated that our algorithms are comparable to other similar methods. In the future, we could consider the following points: (i) Whether we can use a proper line search to determine an appropriate step size, which can reduce the complexity and enhance the accuracy of the algorithm. (ii) Further experiments on the practical problems could be performed in the future to check the performance of the presented algorithms.

Author Contributions: Writing-original draft preparation, H.L.; writing-review and editing, Y.L. and M.Z. All authors have read and agreed to the published version of the manuscript.

Funding: This research was funded by the National Natural Science Foundation of China Grant No.11661009, the High Level Innovation Teams and Excellent Scholars Program in Guangxi institutions of higher education Grant No. [2019]52, the Guangxi Natural Science Key Fund No. 2017GXNSFDA198046, the Special Funds for Local Science and Technology Development Guided by the Central Government No. ZY20198003, the special foundation for Guangxi Ba Gui Scholars, and the Basic Ability Improvement Project for Young and Middle-Aged Teachers in Guangxi Colleges and Universities No. 2020KY30018.

Conflicts of Interest: The authors declare no conflict of interest.

\section{References}

1. Robbins, H.; Monro, S. A stochastic approximation method. Ann. Math. Stat. 1951, 22, 400-407. [CrossRef]

2. Chung, K.L. On a stochastic approximation method. Ann. Math. Stat. 1954, 25, 463-483. [CrossRef]

3. Polyak, B.T.; Juditsky, A.B. Acceleration of stochastic approximation by averaging. SIAM J. Control Optim. 1992, 30, 838-855. [CrossRef]

4. Ruszczyǹski, A.; Syski, W. A method of aggregate stochastic subgradients with online stepsize rules for convex stochastic programming problems. In Stochastic Programming 84 Part II; Springer: Berlin/Heidelberg, Germany, 1986; pp. 113-131.

5. Wright, S.; Nocedal, J. Numerical Optimization; Springer: Berlin/Heidelberg, Germany, 1999; Volume 35, p. 7.

6. Bordes, A.; Bottou, L. SGD-QN: Careful quasi-Newton stochastic gradient descent. J. Mach. Learn. Res. 2009, 10, 1737-1754.

7. Byrd, R.H.; Hansen, S.L.; Nocedal, J.; Singer, Y. A stochastic quasi-Newton method for large-scale optimization. SIAM J. Optim. 2016, 26, 1008-1031. [CrossRef]

8. Gower, R.; Goldfarb, D.; Richtárik, P. Stochastic block BFGS: Squeezing more curvature out of data. In Proceedings of the 33rd International Conference on Machine Learning, New York, NY, USA, 19-24 June 2016; Volume 48, pp. 1869-1878.

9. Covei, D.P.; Pirvu, T.A. A stochastic control problem with regime switching. Carpathian J. Math. 2021, 37, 427-440. [CrossRef]

10. Wei, Z.; Li, G.; Qi, L. New quasi-Newton methods for unconstrained optimization problems. Appl. Math. Comput. 2006, 175, 1156-1188. [CrossRef]

11. Durrett, R. Probability: Theory and Examples; Cambridge University Press: Cambridge, UK, 2019.

12. Deng, N.Y.; Li, Z.F. Ome global convergence properties of a conic-variable metric algorithm for minimization with inexact line searches. Numer. Algebra Control Optim. 1995, 5, 105-122.

13. Allen-Zhu, Z.; Hazan, E. Variance reduction for faster non-convex optimization. In Proceedings of the 33rd International Conference on Machine Learning, New York, NY, USA, 19-24 June 2016; Volume 48, pp. 699-707.

14. Shalev-Shwartz, S.; Shamir, O.; Sridharan, K. Learning kernel-based halfspaces with the 0-1 loss. SIAM J. Comput. 2011, 40, 1623-1646. [CrossRef]

15. Ghadimi, S.; Lan, G. Stochastic first-and zeroth-order methods for nonconvex stochastic programming. SIAM J. Optim. 2013, 23, 2341-2368. [CrossRef]

16. Mason, L.; Baxter, J.; Bartlett, P.; Frean, M. Boosting algorithms as gradient descent in function space. Proc. Adv. Neural Inf. Process. Syst. 1999, 12, 512-518.

17. Johnson, R.; Zhang, T. Accelerating stochastic gradient descent using predictive variance reduction. Adv. Neural Inf. Process. Syst. 2013, 26, 315-323.

18. Defazio, A.; Bach, F.; Lacoste-Julien, S. SAGA: A fast incremental gradient method with support for non-strongly convex composite objectives. In Proceedings of the Advances in Neural Information Processing Systems, Montreal, QC, Canada, 8-13 December 2014; pp. 1646-1654. 